Supplementary Information for

\title{
An eco- and user-friendly herbicide
}

Xiangjun Zhang ${ }^{\mathrm{a}}$, Qiaoxian Huang ${ }^{\mathrm{a}}$, Zhe-Ze Zhao, ${ }^{\mathrm{b}}$ Xiaoqiu Xuc ${ }^{\mathrm{c}}$, Shengke $\mathrm{Li}^{\mathrm{a}}$, Hang Yin ${ }^{\mathrm{a}}$, Lanlan $\mathrm{Li}^{\mathrm{c}}$, Jianxiang Zhang ${ }^{\mathrm{c}, *}$ and Ruibing Wang,

${ }^{a}$ State Key Laboratory of Quality Research in Chinese Medicine, Institute of Chinese Medical Sciences, University of Macau, Taipa, Macau 999078, China.

${ }^{\mathrm{b}}$ School of Life Sciences, The Chinese University of Hong Kong, Shatin, New Territories, Hong Kong, China.

${ }^{\mathrm{c}}$ Department of Pharmaceutics, College of Pharmacy, Third Military Medical University, Chongqing 400038, China.

* To whom correspondence should be addressed. Email: jxzhang@tmmu.edu.cn (Jianxiang Zhang); rwang@um.edu.mo (Ruibing Wang). 


\section{This Supplementary Information includes:}

Figure S1. CB[7] reduced the PQ-induced cytotoxicity in vitro in a time-dependent manner Figure S2. H\&E stained sections of intestine from the mice administered with PQ or PQ@CB[7].

Figure S3. Weight changes and organ indices of mice administered with PQ@CB[7] at the PQ dose of $300 \mathrm{mg} / \mathrm{kg}$ and $600 \mathrm{mg} / \mathrm{kg}$.

Figure S4. Organ indices of mice administered with PQ@CB[7] at the PQ dose of 300mg/kg.

Figure S5. H\&E stained sections of intestine from the survived mice administered with PQ@CB[7].

Figure S6. H\&E stained sections of major organs from the survived mice administered with PQ@CB[7].

Figure S7. Top view of the model weed (Zoysia matrella). 


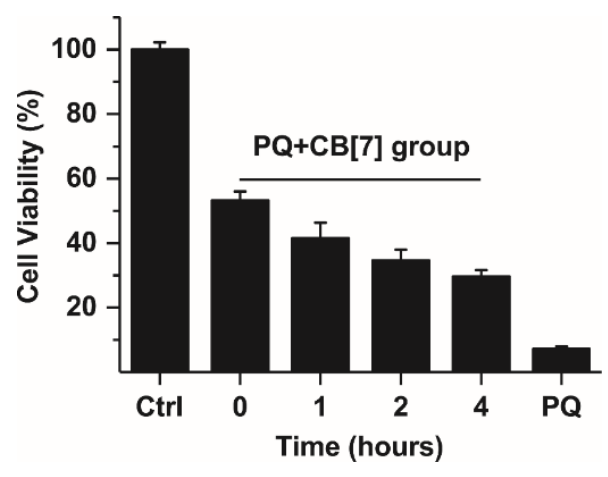

Figure S1. CB[7] reduced the PQ-induced cytotoxicity in a time-dependent manner. RAW264.7 cells were firstly incubated with $0.5 \mathrm{mM}$ PQ and $\mathrm{CB}$ [7] was then added into the medium (the final concentration of $\mathrm{CB}$ [7] is $2 \mathrm{mM}$ ) at $0,1,2$ and $4 \mathrm{~h}$ after PQ. For the PQ-alone group, cells were incubated with $0.5 \mathrm{mM}$ $\mathrm{PQ}$ without any $\mathrm{CB}[7]$ added. Cell viability was then determined at $24 \mathrm{~h}$. 


\section{Control PQ 24h PQ@CB 24h}

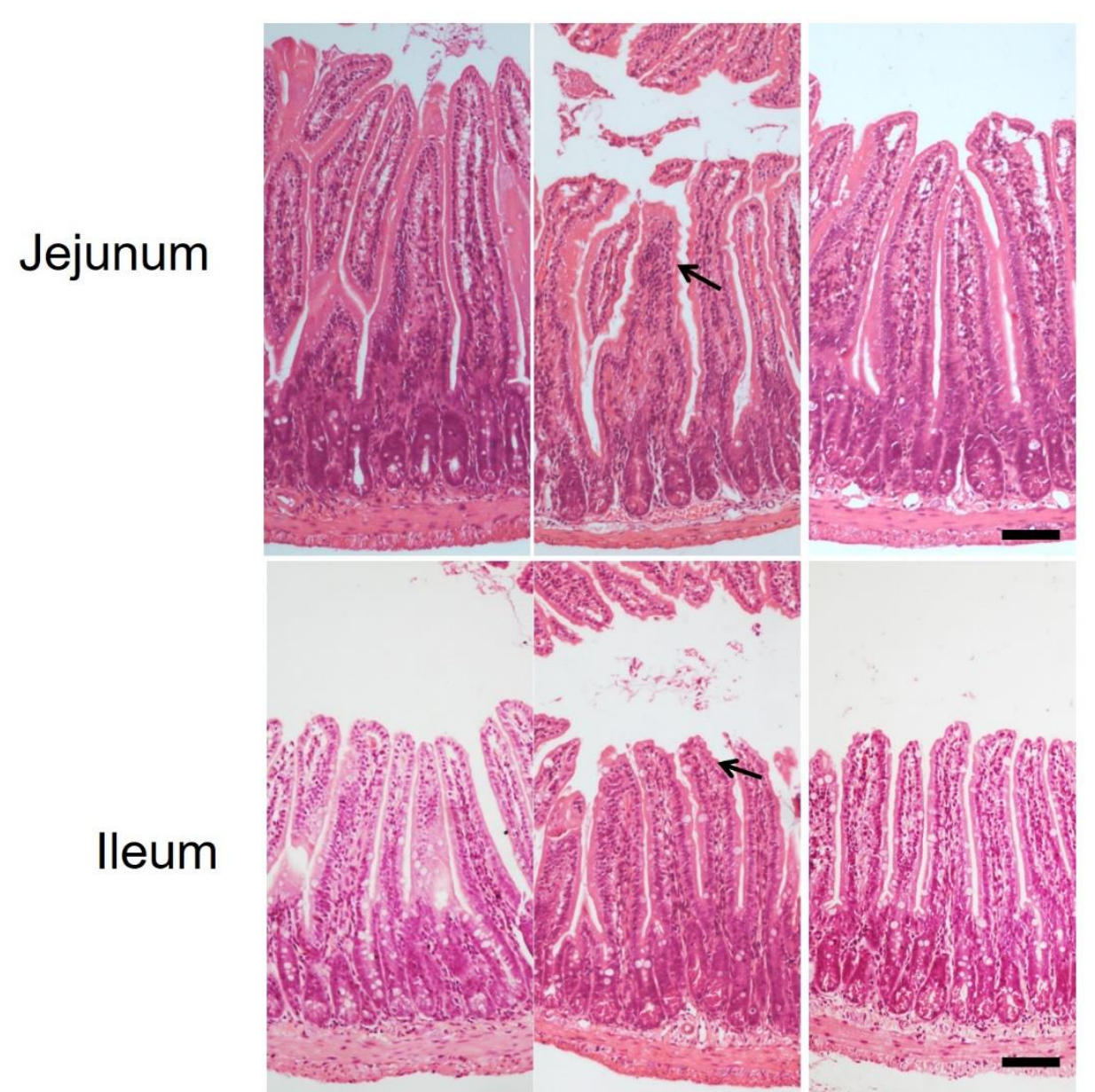

Figure S2. H\&E stained sections of intestine from the mice administered with PQ or PQ@CB[7]. Samples were collected from mice sacrificed 24h after orally administered with PQ or PQ@CB[7] (molar ratio $=1: 2$ ) at the PQ dose $300 \mathrm{mg} / \mathrm{kg}$. Arrows indicated the injuries of intestine. Scale bar $=200 \mu \mathrm{m}$. 

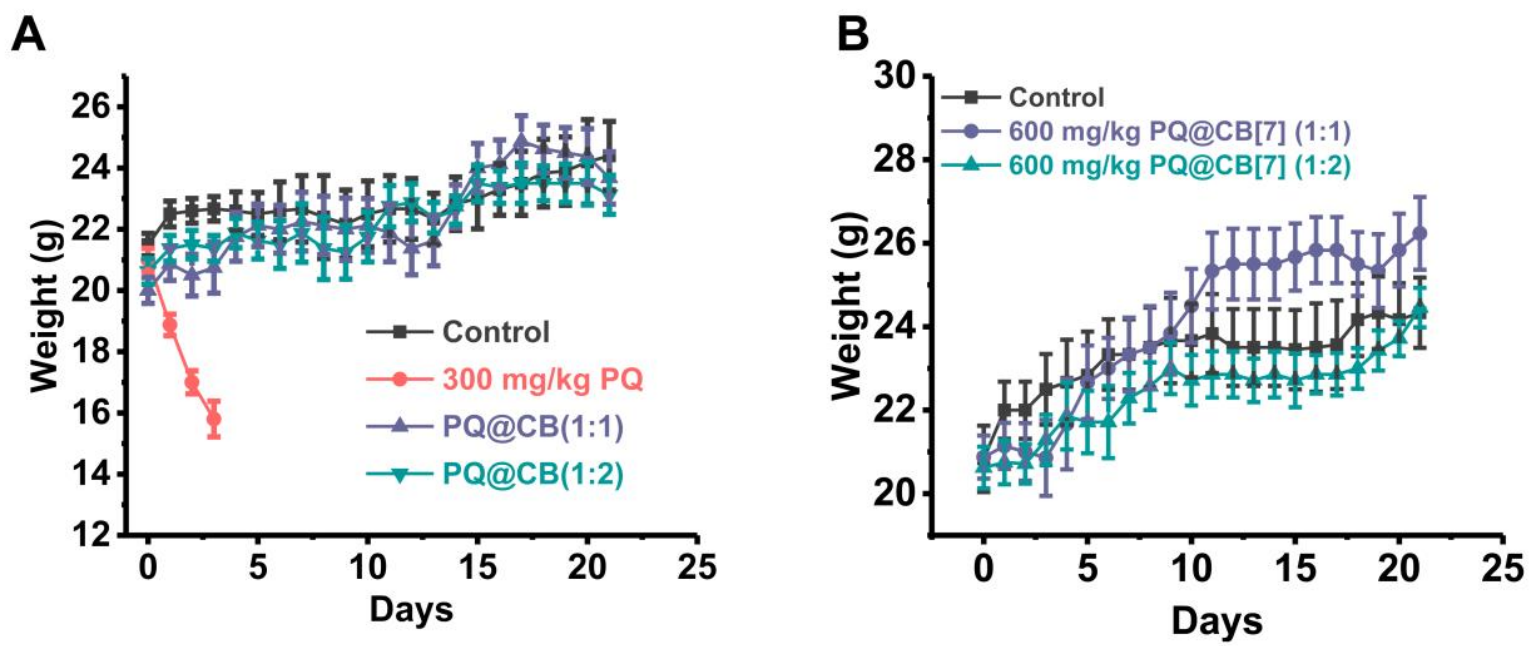

Figure S3. Weight changes and organ indices of mice administered with PQ@CB[7] at the PQ dose of $300 \mathrm{mg} / \mathrm{kg}$ and $600 \mathrm{mg} / \mathrm{kg}$. (A) Weight changes of mice after oral administration with saline (control group, $n=6)$, PQ $(n=8)$ and PQ@ $\mathrm{CB}[7]$ (molar ratio of PQ/CB[7] =1:1 and 1:2, $n=8)$ at the PQ dose of 300 $\mathrm{mg} / \mathrm{kg}$. Data are presented as means \pm SEM. (B) Mice were orally administered with saline (control group, $n$ =6) $\mathrm{PQ} @ \mathrm{CB}$ [7] (molar ratio of PQ/CB[7] = 1:1 and 1:2, $n=8$ ) at the PQ dose of $600 \mathrm{mg} / \mathrm{kg}$. Data are presented as means \pm SEM. 


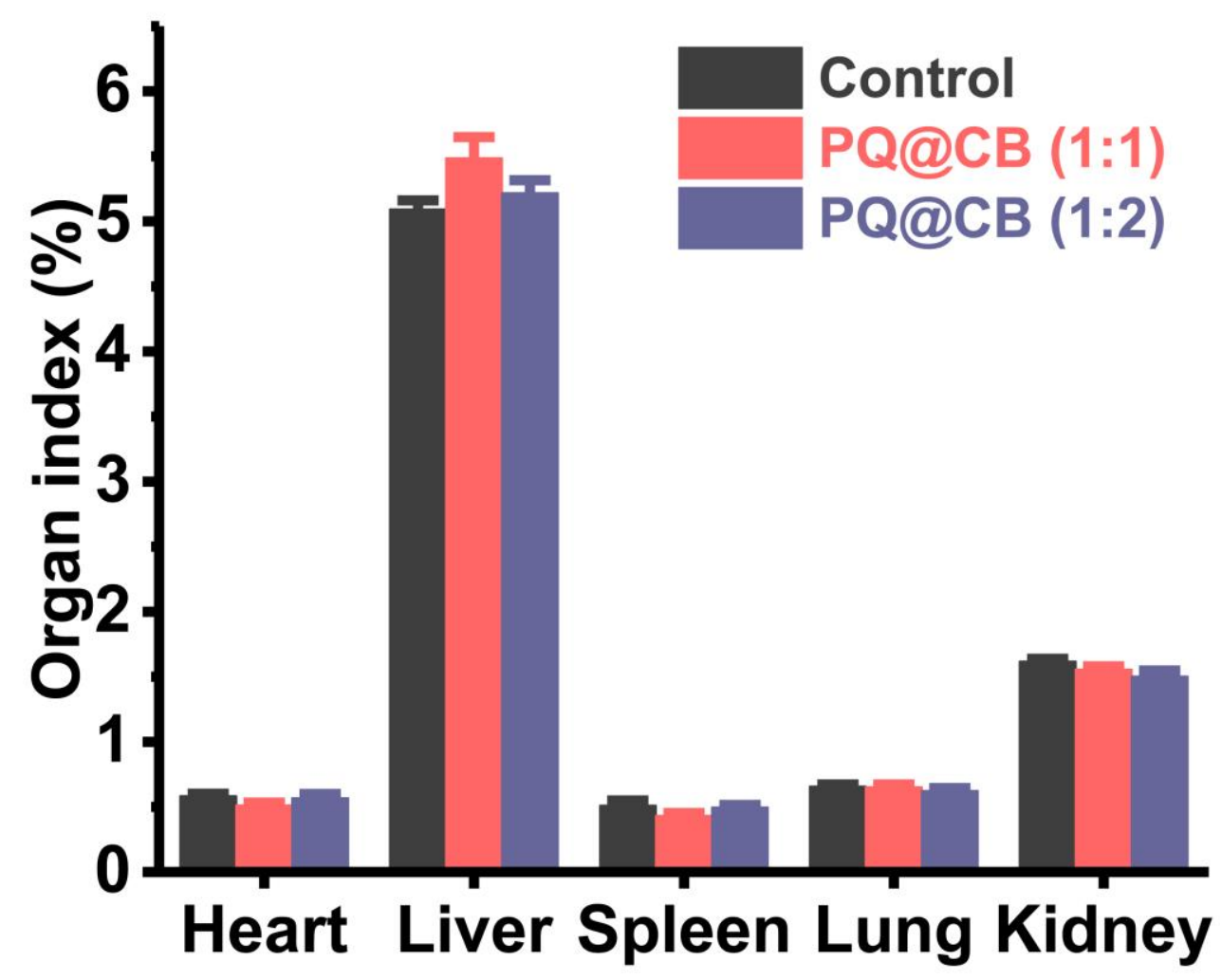

Figure S4. Organ indices of mice administered with PQ@CB[7] at the PQ dose of 300mg/kg. Organ indices of mice at 21 days after orally received with saline (control group, $n=6)$, PQ $(n=8)$ and PQ@CB[7] (molar ratio of $\mathrm{PQ} / \mathrm{CB}[7]=1: 1$ and $1: 2, n=8$ ) at a PQ dose of $300 \mathrm{mg} / \mathrm{kg}$. Data are presented as means \pm SEM. 


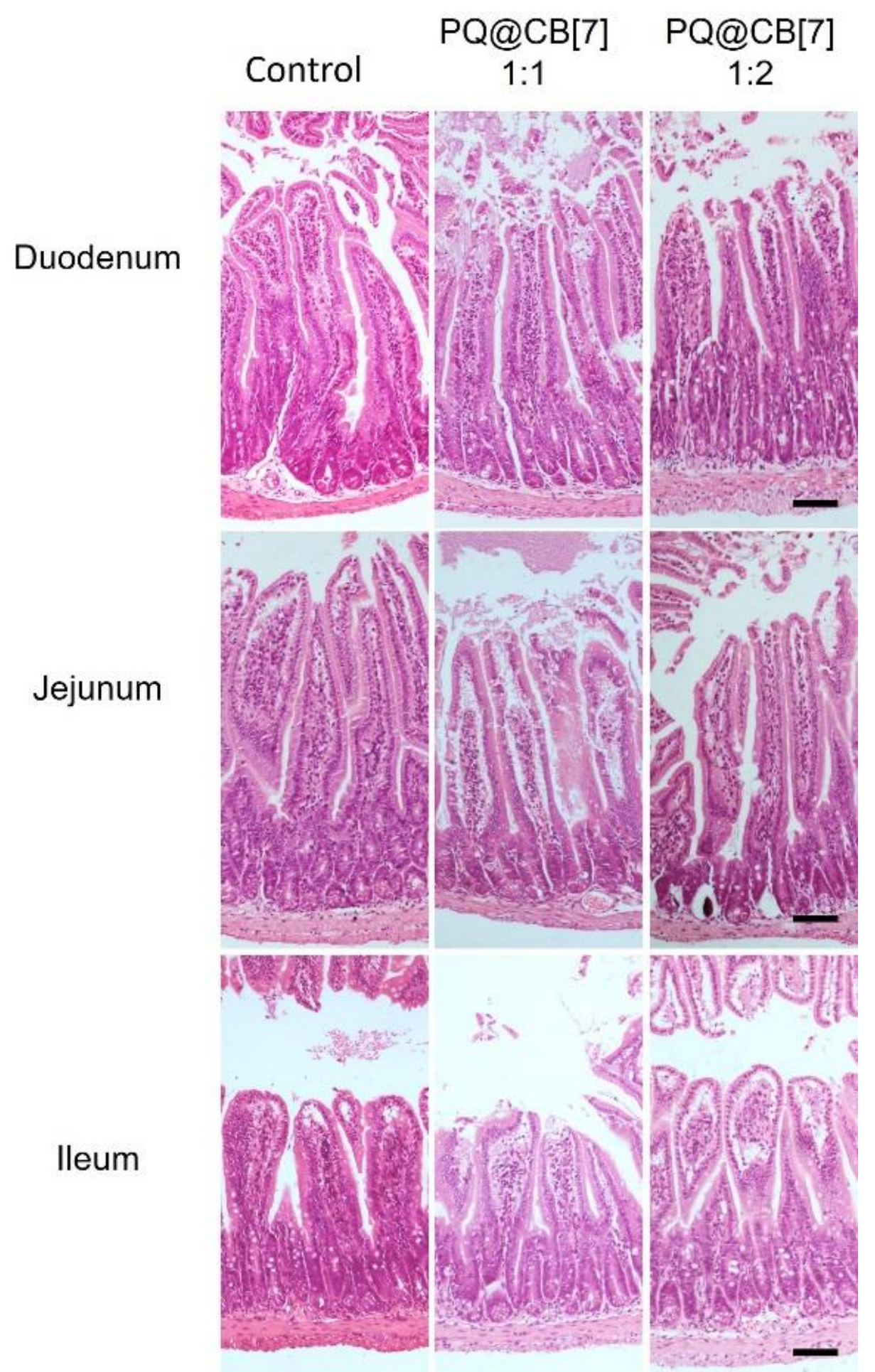

Figure S5. H\&E stained sections of intestine from the survived mice administered with PQ@CB[7]. Samples were collected from mice sacrificed 21days after oral administration with PQ@CB[7] (molar ratio $=1: 1$ or $1: 2$ ) at the PQ dose $300 \mathrm{mg} / \mathrm{kg}$. Scale bar $=200 \mu \mathrm{m}$. 


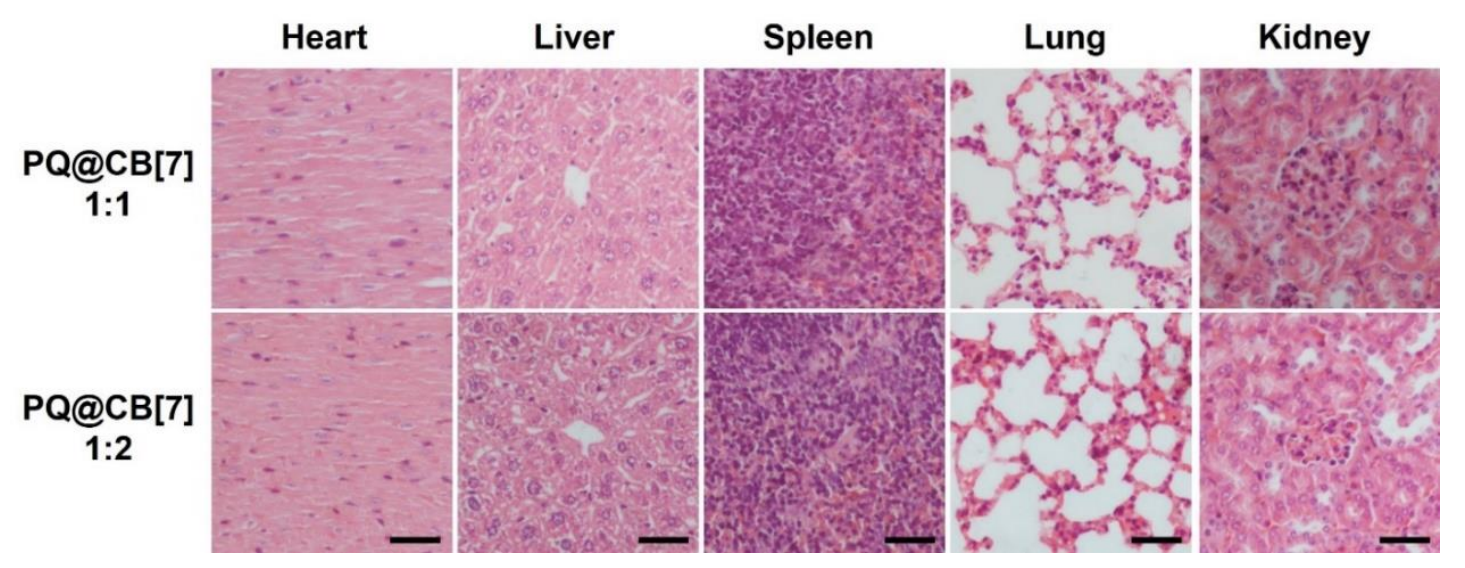

Figure S6. H\&E stained sections of major organs from the survived mice administered with PQ@CB[7]. Samples were collected from mice sacrificed 21 days after oral administration with PQ@CB[7] $($ molar ratio $=1: 1$ or 1:2) at the PQ dose of $300 \mathrm{mg} / \mathrm{kg}$. Scale bar $=200 \mu \mathrm{m}$. 

Control
$P Q$
PQ@CB[7] (1:1)
PQ@CB[7] (1:2)
$\mathrm{CB}[7]$

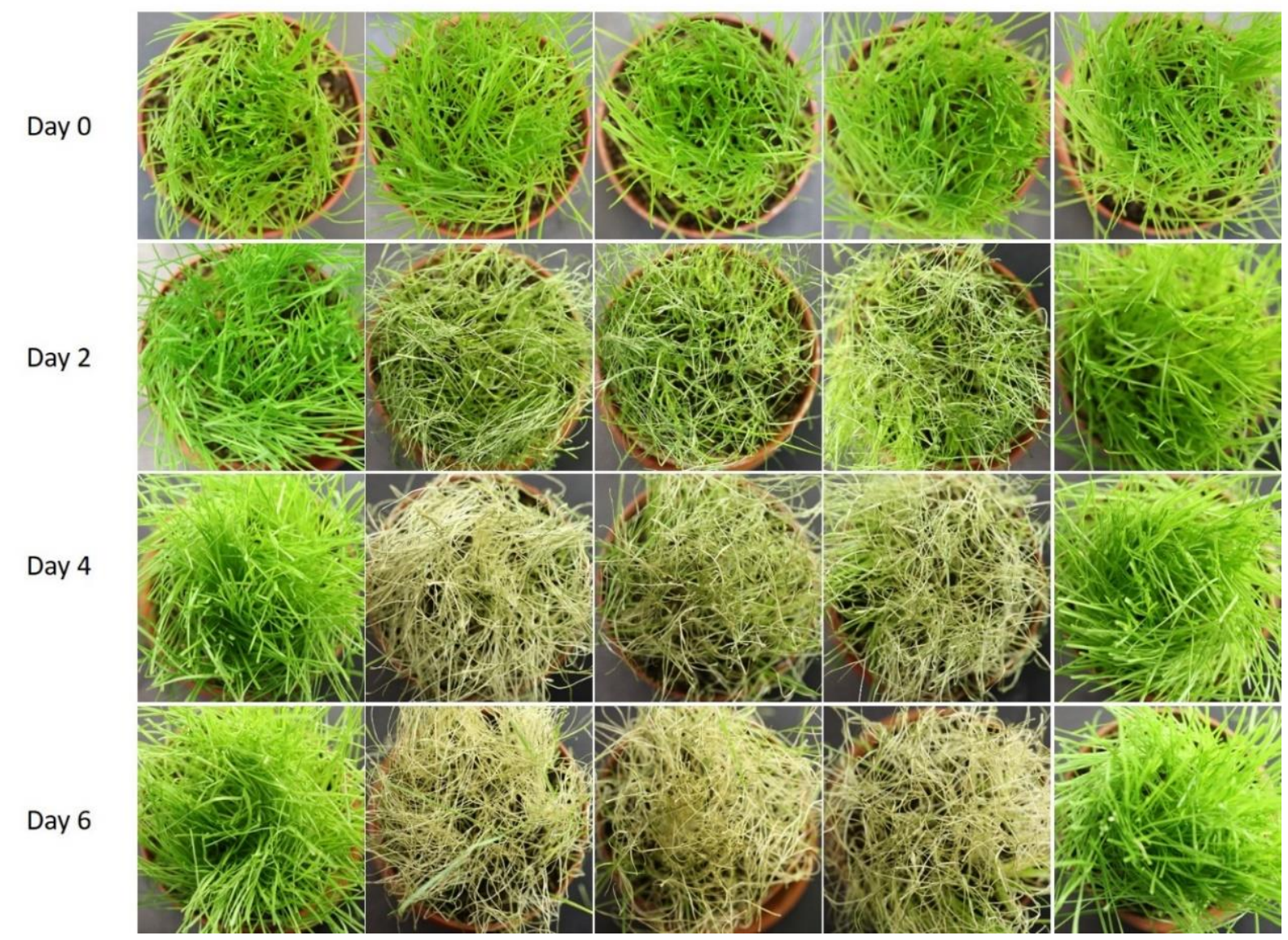

Figure S7. Top view of the model weed (Zoysia matrella). 